\title{
Estudo de estabilidade de equações diferenciais ordinárias autônomas e aplicação
}

Stability study of autonomous ordinary differential equations and application

\author{
Luciano Aparecido Magrini ${ }^{1}$ e Marta Cilene Gadotti ${ }^{2}$ \\ ${ }^{1}$ Instituto Federal de Educação, Ciência e Tecnologia - Campus São Paulo, Brasil \\ luciano.magrini@hotmail.com \\ 2Universidade Estadual Paulista "Julio de Mesquita Neto" - Campus Rio Claro \\ martacg@rc.unesp.br
}

\begin{abstract}
Resumo
Pretende-se com este trabalho introduzir alguns resultados importantes sobre estabilidade de soluções de equações diferenciais ordinárias autônomas e utilizá-los no estudo de estabilidade de um modelo matemático do controle biológico de pragas da broca da cana.

Palavras-chave: sistema linear, equação autônoma, estabilidade, autovalores, exponencial matricial.
\end{abstract}

\begin{abstract}
The aim of this work is to introduce some important results on stability of solutions of autonomous ordinary differential equations and use them in the stability study of a mathematical model of the biological pest control of the sugarcane borer.
\end{abstract}

Keywords: linear systems, autonomous equation, stability, eigenvalues, matrix exponential. 


\section{Introdução}

As equações diferenciais ordinárias (EDO) são indispensáveis na modelagem de fenômenos presentes nas mais diversas áreas do conhecimento, em que o tempo deve ser admitido como grandeza contínua. A análise dos modelos depende de dados coletados de maneira experimental e que, portanto, estão sujeitos a erros de medição ou coleta, por exemplo.

Particularmente espera-se que as soluções de uma EDO possam auxiliar no entendimento do fenômeno em estudo, apesar de pequenas perturbações nos dados experimentais. Porém determinar explicitamente as soluções de qualquer equação diferencial é uma tarefa árdua devido a alta complexidade da maior parte das equações diferenciais envolvidas no processo de modelagem. Portanto o estudo de propriedades qualitativas de soluções de equações diferenciais tem um papel fundamental no desenvolvimento desta análise. Um fenômeno qualitativo de grande interesse é a noção de estabilidade de uma certa solução de uma equação diferencial, a qual será discutida neste trabalho.

Na próxima seção serão desenvolvidos alguns resultados para sistema autônomos com respeito à estabilidade. Na seção 3 será apresentado um modelo matemático do controle biológico de pragas da broca da cana, baseado na referência [RAFIKOV, 2012] e analisada a estabilidade de seus pontos de equilíbrio. Também serão apresentadas algumas considerações e sugestões sobre $o$ trabalho docente com modelos biológicos na Educação Básica.

\section{Fatos básicos}

Seja $F: D \rightarrow \mathbb{R}^{n}$ uma função contínua num aberto $D \subset \mathbb{R} \times \mathbb{R}^{n}$ e admita que suas derivadas parciais $\frac{\partial F}{\partial x_{i}}$, com $i=1,2, \ldots, n$ existam e também sejam contínuas em $D$. Uma equação diferencial de primeira ordem é dada pela relação

$$
X^{\prime}=F(t, X),
$$

em que $X(t)=\left(x_{1}(t), \ldots x_{n}(t)\right)$ é a função incógnita.

Um sistema linear homogêneo de primeira ordem pode ser escrito na forma:

$$
\left\{\begin{array}{c}
x_{1}^{\prime}(t)=a_{11}(t) x_{1}(t)+a_{12}(t) x_{2}(t)+\ldots+a_{1 n}(t) x_{n}(t) \\
x_{2}^{\prime}(t)=a_{21}(t) x_{1}(t)+a_{22}(t) x_{2}(t)+\ldots+a_{2 n}(t) x_{n}(t) \\
\vdots \\
x_{n}^{\prime}(t)=a_{n 1}(t) x_{1}(t)+a_{n 2}(t) x_{2}(t)+\ldots+a_{n n}(t) x_{n}(t),
\end{array}\right.
$$

onde $a_{i j}$ são funções contínuas definidas em algum intervalo $I$ da reta real, $i=1,2, \ldots n$.

Quando as funções $a_{i i}$ são constantes o sistema (2) em notação matricial, pode ser representado por:

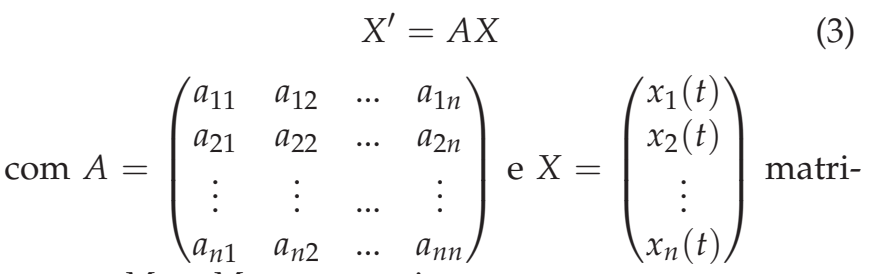
zes em $M_{n}$ e $M_{1 \times n}$ respectivamente.

Dada uma condição inicial $\left(t_{0}, x_{0}\right) \in \mathbb{R}^{1+n}$, a existência e a unicidade de solução da equação (3) estão garantidas pelas hipóteses feitas acerca da função $F$ no início da seção. Denotaremos por $\gamma\left(t, t_{0}, x_{0}\right)$ a solução de (3) que em $t_{0}$ vale $x_{0}$

Uma solução $\gamma\left(t, t_{0}, x_{0}\right)$ de (2) é chamada de estável se dado $\epsilon>0$ existir $\delta>0$ tal que se $\left\|x_{0}-\bar{x}_{0}\right\|<$ $\delta$ então $\left\|\gamma\left(t, t_{0}, x_{0}\right)-\gamma\left(t, t_{0}, \bar{x}_{0}\right)\right\|<\epsilon$ para $t>t_{0}$. Se $\gamma\left(t, t_{0}, x_{0}\right)$ for estável e existir $\alpha>0$ tal que se $\| x_{0}-$ $\bar{x}_{0} \|<\alpha$ então $\left\|\gamma\left(t, t_{0}, x_{0}\right)-\gamma\left(t, t_{0}, \bar{x}_{0}\right)\right\| \rightarrow 0$ quando $t \rightarrow$ $\infty$ então a solução será chamada de assintoticamente estável. Caso uma solução não seja estável, dizemos que ela é instável.

Os pontos $\bar{X} \in \mathbb{R}^{n}$ tais que $A \bar{X}=0$ são chamados de pontos de equilíbrio da equação (3). Para estudar a estabilidade da equação (3), basta que analisemos a estabilidade dos pontos $\bar{X}$, veja a referência [BRAUN, 1979].

A estabilidade da equação (3) é determinada pela análise dos autovalores da matriz $A$. Para demonstrarmos este resultado, usaremos o lema abaixo, cuja prova pode ser encontrada em [BARREIRA, 2012].

Lema 1: Suponha que a matriz $A \in M_{n}$ possua apenas autovalores com parte real negativa; então devem existir constantes $k, \alpha>0$ tais que para $t \geq 0$

$$
\left\|e^{A t}\right\| \leq k e^{-\alpha t}
$$

com a norma $\|A\|=\sup _{x \neq 0} \frac{\|A(X)\|}{\|X\|}$ definida a partir de qualquer norma $\|$.$\| em \mathbb{R}^{n}$.

Teorema 1: A solução de equilíbrio $X=0$ da equação $X^{\prime}=A X$ linear e homogênea é:

1. Assintoticamente Estável se todos os autovalores de $A$ possuírem parte real negativa;

2. Estável se todos os autovalores de $A$ possuírem parte real negativa ou parte real nula e os autovalores de parte real nula possuírem multiplicidade algébrica igual à multiplicidade geométrica;

3. Instável se pelo menos um dos autovalores tiver parte real positiva ou se existir algum autovalor com parte real nula com multiplicidade algébrica maior que a multiplicidade geométrica. 
Demonstração. Provemos apenas o item 1. O restante da demonstração pode ser encontrada em [BRAUN, 1979].

Como toda solução $X(t)=\left(X_{1}(t), \ldots X_{n}(t)\right)^{T}$ de (3) é da forma $X(t)=e^{A t} X_{0}$, seja $\phi_{i j}(t)$ o elemento $i j$ da matriz $e^{A t}$ e seja $X_{0}=\left(x_{1}^{0}, \ldots, x_{n}^{0}\right)^{T} \in \mathbb{R}^{n}$; então

$$
X_{i}(t)=\phi_{i 1}(t) x_{1}^{0}+\ldots+\phi_{\text {in }}(t) x_{n}^{0} .
$$

Suponha que todos os autovalores de $A$, denominados $\lambda_{1}, \ldots \lambda_{r}$, possuam parte real negativa, ou seja, que $\max _{i=1, \ldots, r} \operatorname{Re}\left(\lambda_{i}\right)<0$. Seja $-\alpha_{1}=\max _{i=1, \ldots, r} \operatorname{Re}\left(\lambda_{i}\right)$ o valor da maior parte real no conjunto dos autovalores. Então, existem pelo Lema $1, K>0$ e $\alpha>0$ tais que $-\alpha_{1}<-\alpha<0$ e $\left|\phi_{i j}(t)\right| \leq K e^{-\alpha t}$ para todo $t \geq 0$. Consequentemente, para todo $i=1, \ldots, n$ temos

$$
\begin{aligned}
\left|X_{i}(t)\right| & =\left|\phi_{i 1}(t) x_{1}^{0} \ldots+\phi_{i n}(t) x_{n}^{0}\right| \\
& \leq K e^{-\alpha t}\left(\left|x_{1}^{0}\right|+\ldots+\left|x_{n}^{0}\right|\right) \\
\leq & K e^{-\alpha t} \max \left\{\left|x_{1}^{0}\right|, \ldots,\left|x_{n}^{0}\right|\right\}=K e^{-\alpha t} n\left\|X_{0}\right\| .
\end{aligned}
$$

Assim,

$$
\|X(t)\|=\max \left\{\left|X_{1}(t)\right|, \ldots,\left|X_{n}(t)\right|\right\} \leq n K e^{-\alpha t}\left\|X_{0}\right\| .
$$

Dado $\epsilon>0$, para garantir $\|X(t)\|<\epsilon$ é suficiente que ocorra $\left\|X_{0}\right\|<\frac{\epsilon}{n K}$, com $t=0$. Neste caso, escolhe$\operatorname{mos} \delta>0$ tal que $\delta<\frac{\epsilon}{n K}$, de onde $\left\|X_{0}\right\| \leq \delta$ e portanto, $\|X(t)\|<\epsilon$. Resulta também que para qualquer condição inicial temos $\|X(t)\| \rightarrow 0$ quando $t \rightarrow+\infty$, ou seja, a solução de equilíbrio é assintoticamente estável.

O estudo de sistemas não lineares da forma $X^{\prime}=$ $F(X)$ pode ser realizado inicialmente calculando-se os pontos de equilíbrio e em seguida analisando o comportamento próximo a esses pontos. É possível provar que o comportamento local do sistema não linear autônomo próximo a um ponto de equilíbrio hiperbólico $X_{0}$ (isto é, nenhum autovalor da matriz $A=D F\left(X_{0}\right)$ tem parte real nula) é qualitativamente determinado pelo comportamento do sistema linear $X^{\prime}=A X$, em que $A=D F\left(X_{0}\right)$, próximo à origem. A função $A X=D F\left(X_{0}\right) X$ é chamada de parte linear de $F$ em $X_{0}$. Para maiores detalhes veja o Teorema de Hartman-Grobman na referência [PERKO, 1996], por exemplo. A seguir apresentaremos os resultados sobre sistemas não lineares autônomos necessários para o desenvolvimento da seção 3.

Consideremos a equação $X^{\prime}=F(X)$, em que $F: U \subset$ $\mathbb{R}^{n} \longrightarrow \mathbb{R}^{n}$ é uma função não linear e denotemos por $\bar{X}$ um ponto de equilíbrio.

O lema abaixo, cuja demonstração essencialmente usa o Polinômio de Taylor, será fundamental para as considerações que faremos na sequência.

Lema 2: Seja $F: U \subset \mathbb{R}^{n} \rightarrow \mathbb{R}^{n}$ uma função com derivadas parciais de segunda ordem contínuas com respeito a cada uma de suas variáveis $x_{1}, x_{2}, \ldots, x_{n}$ e seja $X$ um ponto qualquer do aberto $U$ (em particular tomaremos $X=\bar{X}$ um ponto de equilíbrio de $F$ ). Então, dado $Z=\left(z_{1}, z_{2}, \ldots, z_{n}\right) \in \mathbb{R}^{n}$ suficientemente próximo de $\bar{X}$, $F(\bar{X}+Z)$ pode ser escrita como

$$
F(\bar{X}+Z)=F(\bar{X})+D F(\bar{X}) Z+G(Z),
$$

onde

$$
\frac{G(Z)}{\max \left\{\left|z_{1}\right|,\left|z_{2}\right|, \ldots,\left|z_{n}\right|\right\}}
$$

é uma função contínua na variável $Z$ e identicamente nula se $Z=0$ e

$$
D F(\bar{X})=\left(\begin{array}{ccc}
\frac{\partial F_{1}(\bar{X})}{\partial x_{1}} & \ldots & \frac{\partial F_{1}(\bar{X})}{\partial x_{n}} \\
\vdots & \ddots & \vdots \\
\frac{\partial F_{n}(\bar{X})}{\partial x_{1}} & \cdots & \frac{\partial F_{n}(\bar{X})}{\partial x_{n}}
\end{array}\right)
$$

é a matriz jacobiana de $F$ calculada no ponto $\bar{X}$.

A estabilidade do ponto de equilíbrio $\bar{X}$ é determinada pela análise da matriz $D F(\bar{X})$, nos mesmos moldes do que fizemos para equações lineares. Para provarmos isso, assumiremos sem perda de generalidade que $\bar{X}=0$ é a solução de equilíbrio. De fato, para um ponto $\bar{X}$ qualquer de equilíbrio, considere $Z=X-\bar{X}$ e portanto $X=Z+\bar{X}$. Segue que $Z^{\prime}=F(X)=F(Z+\bar{X})$ ou ainda $Z^{\prime}=G(Z)$. Se $\bar{X}$ é a solução de equilíbrio de $X^{\prime}=F(X)$, isto é, se $F(\bar{X})=0$ então 0 é solução de equilíbrio de $Z^{\prime}=G(Z)$ pois $G(0)=F(0+\bar{X})=0$.

Os dois lemas a seguir serão utilizados na demonstração do resultado sobre a estabilidade das equações não lineares. Para a demonstração, consulte as referências indicadas no final do artigo.

Lema 3: (Fórmula da Variação das Constantes) Seja $G: \mathbb{R} \longrightarrow \mathbb{R}^{d}$ uma função contínua. Supondo $\left(t_{0}, X_{0}\right) \in$ $\mathbb{R}^{1+d}$ como condição inicial, a solução da equação $X^{\prime}=$ $A X+G(t)$ é dada por

$$
X(t)=e^{A\left(t-t_{0}\right)} X_{0}+\int_{t_{0}}^{t} e^{A(t-s)} G(s) d s, \quad \text { com } \quad t \in I \subset \mathbb{R} .
$$

Lema 4: (Lema de Gronwall) Sejam $u, v:[a, b] \longrightarrow \mathbb{R}$ funções contínuas com $v(t) \geq 0$ para todo $t \in[a, b]$ e seja $c \in \mathbb{R}$. Se

$$
u(t) \leq c+\int_{a}^{t} u(s) v(s) d s
$$

para qualquer $t \in[a, b]$, então

$$
u(t) \leq c e^{\int_{a}^{t} v(s) d s}
$$

para qualquer $t \in[a, b]$.

Teorema 2: Considere $X^{\prime}=F(X)$ e suponha que $F$ possua derivadas de segunda ordem contínuas. Então: 
1. O ponto de equilíbrio $\bar{X}$ de $X^{\prime}=F(X)$ é assintoticamente estável se todos os autovalores da matriz $D F(\bar{X})$ possuem parte real negativa;

2. O ponto de equilíbrio $\bar{X}$ de $X^{\prime}=F(X)$ é instável se pelo menos um dos autovalores de $D F(\bar{X})$ possuir parte real positiva.

Demonstração. Sem perda de generalidade, vamos supor que $\bar{X}=0$ seja o ponto de equilíbrio de $X^{\prime}=F(X)$. Como $X^{\prime}=A X+G(t)$ e $G$ é contínua, segue do Lema 3 e de $X(0)=X_{0}$ que

$$
X(t)=e^{A t} X_{0}+\int_{0}^{t} e^{A(t-s)} G(s) d s, t \in I \subset \mathbb{R} .
$$

Sob as hipóteses assumidas no item 1., não somente $\|X(t)\|$ é limitada para qualquer $t$ como também que $\|X(t)\| \rightarrow 0$ quando $t \rightarrow+\infty$. Pela construção feita na demonstração do Lema 1 sabemos que, sendo os autovalores todos negativos, existem $K>0$ e $k>0$ tais que

$$
\left\|e^{A t} X_{0}\right\| \leq K e^{-k t}\left\|X_{0}\right\|, \forall t \in I
$$

de onde resulta também

$$
\left\|e^{A(t-s)} G(t)\right\| \leq K e^{-k(t-s)}\|G(t)\|, \forall t \in I .
$$

Além disso, existe $\sigma>0$ tal que

$$
\|X\|<\sigma \Rightarrow\|G(t)\| \leq \frac{k}{2 K}\|X\| .
$$

Portanto, podemos agora escrever para $\|X\|<\sigma$ :

$$
\begin{gathered}
\|X(t)\| \leq\left\|e^{A t} X_{0}\right\|+\int_{0}^{t}\left\|e^{A(t-s)} G(s)\right\| d s \\
\|X(t)\| \leq K e^{-k t}\left\|X_{0}\right\|+\int_{0}^{t} K e^{-k(t-s)}\|G(s)\| d s \\
\|X(t)\| \leq K e^{-k t}\left\|X_{0}\right\|+\int_{0}^{t} K e^{-k(t-s)} \frac{k}{2 K}\|X(s)\| d s \\
\|X(t)\| \leq K e^{-k t}\left\|X_{0}\right\|+\frac{k}{2 K} \int_{0}^{t} K e^{-k(t-s)}\|X(s)\| d s .
\end{gathered}
$$

Multiplicando a equação anterior por $e^{k t}$ segue que

$$
e^{k t}\|X(t)\| \leq K\left\|X_{0}\right\|+\frac{k}{2 K} \int_{0}^{t} K e^{k s}\|X(s)\| d s
$$

e fazendo $Z(t)=e^{k t}\|X(t)\|$ a desigualdade acima pode ser colocada na forma

$$
Z(t) \leq K\left\|X_{0}\right\|+\frac{k}{2} \int_{0}^{t} Z(s) d s .
$$

Pelo Lema 4 segue que

$$
Z(t) \leq K\left\|X_{0}\right\| e^{\frac{t k}{2}}
$$

e portanto $Z(t)=e^{k t}\|X(t)\| \leq K\left\|X_{0}\right\| e^{\frac{t k}{2}}$, ou seja,

$$
\|X(t)\| \leq K\left\|X_{0}\right\| e^{-k t} e^{\frac{t k}{2}}=K\left\|X_{0}\right\| e^{-\frac{k}{2} t},
$$

desde que $\|X(s)\| \leq \sigma, 0 \leq s \leq t$. Mas se $\left\|X_{0}\right\|=$ $\|X(0)\|<\frac{\sigma}{K}$ a desigualdade $\|X(t)\| \leq K\left\|X_{0}\right\| e^{-\frac{k}{2} t}$ garante que $\|X(t)\|<\sigma$ se verifica para todo $t$ e consequentemente ela é verdadeira para todo $t$ se o valor inicial estiver sujeito à $\|X(0)\|<\frac{\sigma}{K}$. Neste caso se verifica também $\|X(t)\| \rightarrow 0$, quando $t \rightarrow \infty$.

Para a demonstração do item 2, consulte [PERKO, 1996].

Vale mencionar que o estudo de estabilidade abordado neste trabalho não é único, existem outros métodos importantes para o estudo de estabilidade de ponto de equilíbrio, por exemplo o uso de uma função auxiliar apropriada, este método é conhecido como o segundo método de Liapunov; para maiores detalhes veja as referências [BOYCE, 2002] e [PERKO, 1996].

\section{Análise de Modelo Biológico}

Passamos a descrever nesta seção um modelo de interação entre a broca da cana-de-açucar e seu parasitóide Trichogramma galloi, proposto por [RAFIKOV, 2012]. Introduziremos uma hipótese adicional, de modo a modificar ligeiramente o modelo original e analisaremos sua estabilidade usando a teoria apresentada anteriormente.

Como primeira hipótese para o modelo, admitiremos que as principais fases do desenvolvimento da broca da cana-de-açucar sejam os estágio de "ovo"e "larval". Admitiremos também que o controle biológico da praga é feito através da introdução de um único parasitóide (dos ovos) no ambiente comum, de modo que a interação entre eles não seja modificada pela presença de indivíduos estranhos.

A taxa de infestação dos ovos é suposta proporcional ao número de encontros entre parasita e hospedeiro. Neste caso, supondo $\gamma$ a taxa de parasitismo e chamando de $x$ e $z$ respectivamente o número de indivíduos não infectados e o número de parasitóides, a expressão matemática para a taxa de infestação é

$$
\gamma x z \text {. }
$$

Usaremos ainda o fato de que o parasitóide adulto infecta os ovos da praga no início de sua vida, de modo que o parasitismo no tempo $t$ é causado somente pelos parasitóides que emergiram no tempo $t$, ou seja,

$$
z=\delta n_{2} x_{2}
$$

onde $\delta, n_{2}$ e $x_{2}$ indicam o número de parasitóides que emergem para cada ovo parasitados, a fração de ovos 
parasitados que emergem no tempo $t$ e o número de ovos parasitados no tempo $t$ respectivamente.

Seja $x_{1}$ a população de ovos existentes no instante t. Supondo um crescimento logístico (com capacidade de suporte $K$ ) com taxa de reprodução $\beta$, admitindo a hipótese (7) e notando que $m_{1}$ e $n_{1}$ indicam as taxas de mortalidade desta população e a fração de ovos que emergem no tempo $t$, a variação de $x_{1}$ em relação ao tempo $t$ é dada por

$$
\frac{d x_{1}}{d t}=\beta\left(1-\frac{x_{1}}{K}\right) x_{1}-m_{1} x_{1}-n_{1} x_{1}-\gamma x_{1} z .
$$

Seja agora $x_{2}$ a população de ovos parasitados no instante $t$. Novamente admitindo (7) e supondo $z$ o número de parasitas adultos no ambiente, temos para a variação de $x_{2}$ com relação ao tempo:

$$
\frac{d x_{2}}{d t}=\gamma x_{1} z-m_{2} x_{2}-n_{2} x_{2}
$$

onde as constantes $m_{2}$ e $n_{2}$ representam a taxa de mortalidade de ovos parasitados e fração dos ovos parasitados que emergem em $t$ respectivamente.

Considere agora $x_{3}$ a população de larvas no tempo t. Admitindo $m_{3}$ como taxa de mortalidade das larvas e $n_{3}$ como a fração da população que atinge o estágio de pupa, temos:

$$
\frac{d x_{3}}{d t}=n_{1} x_{1}-m_{3} x_{3}-n_{3} x_{3}
$$

Modificaremos aqui o modelo original de [RAFIKOV, 2012] admitindo uma infestação pela broca da cana-deaçucar mais resistente, supondo que a taxa de mortalidade da broca no estágio larval observada em uma infestação específica é na prática desprezível, de modo que todos os ovos atinjam o estágio larval. Das equações (9), (10) e (11) (onde $m_{3}=0$ e $n_{3}=1$, pela hipótese introduzida), substituindo (8) e tomando $\alpha=\gamma \delta n_{2}$, podemos obter o seguinte sistema (não linear) de equações diferenciais para o modelo de interação entre a broca da cana-de-açúcar e seu parasitóide:

$$
\left\{\begin{array}{c}
\frac{d x_{1}}{d t}=\beta\left(1-\frac{x_{1}}{K}\right) x_{1}-m_{1} x_{1}-n_{1} x_{1}-\alpha x_{1} x_{2}, \\
\frac{d x_{2}}{d t}=\alpha x_{1} x_{2}-m_{2} x_{2}-n_{2} x_{2}, \\
\frac{d x_{3}}{d t}=n_{1} x_{1}-x_{3} .
\end{array}\right.
$$

Observamos que a matriz jacobiana de (12) é dada por

$$
J=\left(\begin{array}{ccc}
\beta\left(1-\frac{2 x_{1}}{K}\right)-m_{1}-n_{1}-\alpha x_{2} & -\alpha x_{1} & 0 \\
\alpha x_{2} & \alpha x_{1}-m_{2}-n_{2} & 0 \\
n_{1} & 0 & -1
\end{array}\right)
$$

Os pontos de equilíbrio de (12) são obtidos através da resolução do sistema algébrico

$$
\left\{\begin{array}{c}
\beta\left(1-\frac{x_{1}}{K}\right) x_{1}-m_{1} x_{1}-n_{1} x_{1}-\alpha x_{1} x_{2}=0, \\
\gamma \alpha x_{1} x_{2}-m_{2} x_{2}-n_{2} x_{2}=0, \\
n_{1} x_{1}-x_{3}=0,
\end{array}\right.
$$

cujas soluções são os pontos

$$
\begin{gathered}
P_{1}=(0,0,0), \\
P_{2}=\left(\frac{K}{\beta}\left(\beta-m_{1}-n_{1}\right), 0, \frac{n_{1} K}{\beta}\left(\beta-m_{1}-n_{1}\right)\right)
\end{gathered}
$$

$\mathrm{e}$

$$
P_{3}=\left(\frac{m_{2}+n_{2}}{\alpha}, \frac{\beta}{\alpha}-\frac{\beta\left(m_{2}+n_{2}\right)}{\alpha^{2} K}-\frac{m_{1}+n_{1}}{\alpha}, \frac{n_{1}\left(m_{2}+n_{2}\right)}{\alpha}\right) .
$$

De fato, por simples inspeção, verifica-se que o ponto $P_{1}=(0,0,0)$ é solução de $(14)$.

Não há sentido biológico supor $x_{1}$ nulo, pois neste caso, estaríamos admitindo a não existência da praga. Portanto, tomemos $x_{1} \neq 0$ e $x_{2}=0$. Da primeira equação de (14), colocando $x_{1}$ em evidência, temos

$$
\beta\left(1-\frac{x_{1}}{K}\right)-m_{1}-n_{1}=0,
$$

de onde segue que

$$
x_{1}=\frac{K}{\beta}\left(\beta-m_{1}-n_{1}\right) .
$$

Como $x_{3}=n_{1} x_{1}$, segue imediatamente da equação anterior que

$$
x_{3}=\frac{n_{1} K}{\beta}\left(\beta-m_{1}-n_{1}\right)
$$

Portanto, $P_{2}=\left(\frac{K}{\beta}\left(\beta-m_{1}-n_{1}\right), 0, \frac{n_{1} K}{\beta}\left(\beta-m_{1}-n_{1}\right)\right)$ também é solução de (14).

Considere $x_{2} \neq 0$. Segue então (da segunda equação do sistema) que

$$
x_{1}=\frac{m_{2}+n_{2}}{\alpha} .
$$

Agora, novamente usando $x_{3}=n_{1} x_{1}$ e a equação anterior, temos

$$
x_{3}=\frac{n_{1}\left(m_{2}+n_{2}\right)}{\alpha} .
$$

Finalmente, substituindo (15) na segunda equação de (14), determinamos $x_{2}$, de modo que uma terceira solução $P_{3}$ é dada por

$P_{3}=\left(\frac{m_{2}+n_{2}}{\alpha}, \frac{\beta}{\alpha}-\frac{\beta\left(m_{2}+n_{2}\right)}{\alpha^{2} K}-\frac{m_{1}+n_{1}}{\alpha}, \frac{n_{1}\left(m_{2}+n_{2}\right)}{\alpha}\right)$. 
Chamamos a atenção para o fato de que é necessário garantir que os pontos encontrados não sejam negativos; ou seja, vamos admitir explicitamente que em $P_{2}$

$$
\beta>m_{1}+n_{1},
$$

e em $P_{3}$, que

$$
\beta>m_{1}+n_{1}-\beta\left(\frac{m_{2}+n_{2}}{k \alpha}\right) .
$$

Passaremos agora à análise da estabilidade de cada um dos pontos de equilíbrio encontrados.

Substituindo as coordenadas de $P_{1}$ em (13), encontramos

$$
J\left(P_{1}\right)=\left(\begin{array}{ccc}
\beta-m_{1}-n_{1} & 0 & 0 \\
0 & -m_{2}-n_{2} & 0 \\
n_{1} & 0 & -1
\end{array}\right),
$$

cujos autovalores são $\lambda_{1}=-1$ e $\lambda_{2}=\beta-m_{1}-n_{1}$ e $\lambda_{3}=-m_{2}-n_{2}$. Vemos portanto, que $P_{1}$ será estável se $\beta<m_{1}+n_{1}$ ou seja, se a taxa de reprodução da população de ovos for menor que a soma das taxas de mortalidade e de transformação de ovos em larvas.

No caso de $P_{2}$, temos:

$J\left(P_{2}\right)=\left(\begin{array}{ccc}\beta\left(1-\frac{2 x_{1}}{K}\right)-m_{1}-n_{1} & -\alpha x_{1} & 0 \\ 0 & \alpha x_{1}-m_{2}-n_{2} & 0 \\ n_{1} & 0 & -1\end{array}\right)$,

cujos autovalores são $\lambda_{1}=-1$ e $\lambda_{2}=\frac{\alpha K\left(\beta-m_{1}-n_{1}\right)}{\beta}-$ $\left(m_{2}+n_{2}\right)$ e $\lambda_{3}=-\beta+m_{1}+n_{1}$.

A negatividade de $\lambda_{3}$ está garantida por (16); para $\lambda_{2}$ ser negativo, devemos ter

$$
\frac{\alpha K\left(\beta-m_{1}-n_{1}\right)}{\beta}-\left(m_{2}+n_{2}\right)<0,
$$

de onde

$$
\beta<\frac{\left(m_{1}+n_{1}\right) K \alpha}{K \alpha+m_{2}+n_{2}} .
$$

Finalmente, substituindo $P_{3}$ em (13), após as devidas simplificações (usamos aqui o fato de que no sistema algébrico (14), na segunda das equações temos $\alpha x_{1}-$ $m_{2}-n_{2}=0$ ) obtemos

$$
J\left(P_{3}\right)=\left(\begin{array}{ccc}
\beta\left(1-\frac{2 x_{1}}{K}\right)-m_{1}-n_{1}-\alpha x_{2} & -\alpha x_{1} & 0 \\
\alpha x_{2} & 0 & 0 \\
n_{1} & 0 & -1
\end{array}\right) .
$$

Um dos autovalores é $\lambda_{1}=-1$; os demais são as raízes da equação

$$
\lambda^{2}+\frac{\beta\left(m_{2}+n_{2}\right)}{\alpha K} \lambda+
$$

$$
\left(\beta-\frac{\beta\left(m_{2}+n_{2}\right)}{\alpha K}-\left(m_{1}+n_{1}\right)\right)\left(m_{2}+n_{2}\right)=0,
$$

que serão negativas se

$$
\left(\beta-\frac{\beta\left(m_{2}+n_{2}\right)}{\alpha K}-\left(m_{1}+n_{1}\right)\right)\left(m_{2}+n_{2}\right)>0,
$$

ou seja, se

$$
\beta>\frac{\left(m_{1}+n_{1}\right) K \alpha}{K \alpha+m_{2}+n_{2}} .
$$

Então, usando os resultados da seção anterior, segue que esta solução também será estável.

Note que não podemos garantir a estabilidade simultânea dos pontos de equilíbrio $P_{2}$ e $P_{3}$ devido às condições (16) e (23). Logo, a análise dos pontos de equilíbrio está concluída.

Quando se admite $t$ como variável discreta as equações que descrevem o fenômeno em estudo não envolvem derivadas; neste caso a criação de modelos matemáticos para fenômenos simples pode ser desenvolvida na Educação Básica, contribuindo para "novo modelo de educação menos alienado e mais comprometido com as realidades dos indivíduos e sociedades" [BASSANEZI, 2002].

Dentre múltiplas possibilidades para a inserção da modelagem matemática na Educação Básica destaca-se a vantagem do estudo de fenômenos biológicos, pois alguns destes ao serem modelados de maneira simplista exigem pouca complexidade matemática e computacional, apresentam um comportamento muito próximo ao observado empiricamente e naturalmente despertam o interesse dos estudantes. Versões simples de problemas como evolução de populações, propagação de doenças, eficácia de campanhas de vacinação, taxa de mortalidade infantil e outros podem ser desenvolvidos. Um exemplo de como é possível desenvolver o tema "evolução de populações"na Educação Básica pode ser encontrado na referência [MAGRINI, 2013].

\section{Conclusões}

Desde a criação do Cálculo, são inúmeras as aplicações e resultados desenvolvidos utilizando as ferramentas deste importante ramo da Matemática. As Equações Diferenciais Ordinárias são objetos matemáticos fundamentais na modelagem de fenômenos físicos, biológicos, ecológicos, etc.

Neste trabalho tivemos a oportunidade de enunciar e provar resultados relevantes sobre o estudo de estabilidade de soluções de equações diferenciais autônomas. O conhecimento sobre o comportamento assintótico das soluções nos fornece uma boa ideia de como elas se comportam no futuro, sem a necessidade de exibir a expressão destas soluções. Vimos também a importância 
de estabelecer resultados para o caso linear, um caso mais simples mas que abrange diversas aplicações, para elaborarmos um estudo de estabilidade sobre o caso não linear, em uma vizinhança do ponto de equilíbrio.

A aplicação envolvida neste trabalho ilustra como essa ferramenta pode ser útil no entendimento de um problema biológico.

Com este trabalho, esperamos que o leitor tenha apreciado um pouco desta bela linha de pesquisa e se aventure aos diversos temas envolvendo as equações diferenciais. São inúmeras as referências - livros e artigos científicos - existentes sobre este tema. Também esperamos que aqueles que são professores da Educação Básica se sintam motivados a criar e investigar modelos matemáticos de fenômenos biológicos em suas versões discretas com seus alunos.

Finalizamos então este trabalho desejando ao leitor interessado uma ótima viagem ao mundo da Matemática, especialmente ao das Equações Diferenciais.

\section{Agradecimentos}

Agradecemos à SBM pela iniciativa na criação do Profmat e à CAPES pelo apoio financeiro. Agradecemos também aos revisores deste artigo pelas sugestões.

\section{Referências}

BARREIRA, L.; VALLS, C. Equações Diferenciais Ordinárias: Teoria Qualitativa. 1a. edição. São Paulo: Livraria da Física, 2012. 260 p.

BASSANEZI, R.C. Ensino-aprendizagem com modelagem matemática: uma nova estratégia. 1a. edição. São Paulo: Contexto, 2002. 392 p.

BOYCE, W.; DIPRIMA, R. C. Equações Diferenciais Elementares e Problemas de Valor de Contorno. 7a. edição Rio de Janeiro: LTC, 2010. 624 p.

BRAUN, M. Equações Diferenciais e suas Aplicações. Rio de Janeiro: Editora Campus, 1979.

MAGRINI, L. A. Modelos Matemáticos e Aplicações ao Ensino Médio. 2013. 126f. Dissertação (Mestrado em Matemática em Rede Nacional) - Instituto de Geociências e Ciências Exatas, UNESP. Rio Claro. 2013.
PERKO, L. Differential Equations and Dynamical Systems. New York: Springer, 1996.

RAFIKOV, M.; LIMEIRA, E.H. Mathematical modelling of the biological pest control of the sugarcane borer, International Journal of Computer Mathematics. vol. 89. no. 3, p. 390-401, 2012 